\title{
Qualidade de vida em pacientes portadores de lesão por pressão
}

\section{Quality of life in patients with pressure injuries}

Calidad de vida en pacientes con lesiones por presión

Grazielle Dafine Fidalgo de Sousa

ORCID: https://orcid.org/0000-0001-7031-7837 Faculdade Estácio de Teresina, Brasil E-mail: sousagrazielle261@gmail.com Marcelo Moura de Carvalho

ORCID: https://orcid.org/0000-0003-3953-4926 Faculdade Estácio de Teresina, Brasil

E-mail: marcelo.mcarvalho@yahoo.com.br

Carylane Nunes Barros

ORCID: https://orcid.org/0000-0003-3742-2269 Faculdade Estácio de Teresina, Brasil E-mail: nunes.carylane@gmail.com

Carlos Weuller Sousa Miguel

ORCID: https://orcid.org/0000-0003-3953-4926 Faculdade Estácio de Teresina, Brasil E-mail: carlos.smiguel02@gmail.com

Cecília Sousa Costa

ORCID: https://orcid.org/0000-0002-7226-6794 Faculdade Estácio de Teresina, Brasil

E-mail:ceciliasc16@gmail.com

Francisca Fabiana Peres Aragão da Silva

ORCID: https://orcid.org/0000-0001-7433-7624 Faculdade Estácio de Teresina, Brasil

E-mail: fabianasoaresperes@outlook.com

Francisco Antônio da Cruz dos Santos

ORCID: https://orcid.org/0000-0003-1065-5695 Faculdade Uniplan Teresina, Brasil E-mail: facs.Francisco.facs@gmail.com

Lauana de Oliveira Lopes

ORCID: https://orcid.org/0000-0002-0602-7437 Faculdade Estácio de Teresina, Brasil E-mail: lauanalopes2000@gmail.com Maria Joelice Barbosa Silva ORCID: https://orcid.org/0000-0003-4238-4199 Faculdade Estácio de Teresina, Brasil E-mail: mariajoelice1@gmail.com Samyla do Nascimento Leite ORCID: https://orcid.org/0000-0003-4001-977X Faculdade Estácio de Teresina, Brasil E-mail: samyla552@gmail.com

Israiane Pereira Mauriz

ORCID: https://orcid.org/0000-0002-9981-9133 Faculdade Estácio de Teresina, Brasil E-mail: israianepm@hotmail.com

\section{Resumo}

Objetivo. O presente trabalho tem como objetivo refletir sobre a qualidade de vida em pacientes portadores de lesão por pressão. Este escrito é de caráter bibliográfico, pois investigou-se em artigos científicos (GIL, 2011) a proposta apresentada para melhor compreensão ao tema de pesquisa. Metodologia. Desse modo, utilizou-se dos seguintes autores a autoras: Portugal e Christovam (2018); Rodrigues e Soriano (2011); Moro e Caliri (2016) entre outras e outros. Resultados. Além disso, por meio da revisão da literatura e sistemática foi possível perceber os entraves vividos por pacientes com lesão por pressão, sua incidência entre outros marcadores que afetam sobremaneira a qualidade de vida destes lesionados (a), assim propor soluções para melhoramento de seu quadro clínico, bem como o compartilhamento de informações para o mesmo e sua família, e isso torna-se possível quando os (a) profissionais da saúde estabelecem um linguagem clara, informativa e objetiva sobre a temática. Nesse sentido, os mesmos (a) devem ter uma formação continuada sobre o tema-gerador, bem como cursos, leituras, debates entre outros meios de trocas 
de conhecimento. Conclusão. Portanto, a qualidade de vida destes é notória quando existe uma equidade da assistência à saúde.

Palavras-chave: Lesão por pressão; Qualidade de vida; Cuidados de enfermagem.

\begin{abstract}
Objective. The present work aims to reflect on the quality of life in patients with pressure injuries. This writing is bibliographical in nature, as the proposal presented was investigated in scientific articles (GIL, 2011) for a better understanding of the research topic. Methodology. Thus, the following authors were used as authors: Portugal and Christovam (2018); Rodrigues and Soriano (2011); Moro and Caliri (2016) among others and others. Results. In addition, through the literature and systematic review, it was possible to perceive the barriers experienced by patients with pressure injuries, their incidence, among other markers that greatly affect the quality of life of these injured people (a), thus proposing solutions to improve their condition. clinical, as well as the sharing of information for the same and their family, and this becomes possible when (a) health professionals establish a clear, informative and objective language on the subject. In this sense, they (a) must have continued training on the theme-generator, as well as courses, readings, debates, among other means of exchanging knowledge. Conclusion. Therefore, their quality of life is notorious when there is equity in health care.
\end{abstract}

Keywords: Pressure injury; Quality of life; Nursing care.

\title{
Resumen
}

Objetivo. El presente trabajo tiene como objetivo reflexionar sobre la calidad de vida de los pacientes con lesiones por presión. Este escrito tiene carácter bibliográfico, ya que la propuesta presentada fue investigada en artículos científicos (GIL, 2011) para una mejor comprensión del tema de investigación. Metodología. Así, se utilizaron como autores a los siguientes autores: Portugal y Christovam (2018); Rodrigues y Soriano (2011); Moro y Caliri (2016) entre otros y otros. Resultados. Además, a través de la literatura y revisión sistemática, fue posible percibir las barreras que experimentan los pacientes con lesiones por presión, su incidencia, entre otros marcadores que afectan en gran medida la calidad de vida de estas personas lesionadas (a), proponiendo así soluciones para mejorar su condición, clínica, así como el intercambio de información para el mismo y su familia, y esto se hace posible cuando (a) los profesionales de la salud establecen un lenguaje claro, informativo y objetivo sobre el tema. En este sentido, deben (a) tener una formación continua en el tema generador, así como cursos, lecturas, debates, entre otros medios de intercambio de conocimientos. Conclusión. Por tanto, su calidad de vida es notoria cuando existe equidad en la atención de la salud.

Palabras clave: Lesión por presión; Calidad de vida; Cuidado de enfermera.

\section{Introdução}

A pele é o maior órgão do corpo humano. Representa cerca de $10 \%$ a $15 \%$ do peso corporal, dividida em camadas que vão da parte mais superficial chamada epiderme, passando por uma camada secundária, derme e chegando a camada mais profunda, hipoderme, apreendendo sinais externos que atingem posteriormente tecido muscular e nervoso. Apresenta - se como um sistema primário de defesas do organismo exercendo inúmeras funções de proteção contra traumas físicos ou térmicos, invasão microbiana, radiação e excreção (Ascari et al., 2014).

De acordo com Macedo (2018,p.3), ter uma boa pele é questão de saúde, ela faz ponte entre o corpo e o meio ambiente, dessa forma, desempenha ainda funções sensoriais, que vão desde a percepção corpórea à captação de estímulos do meio, sendo um grande responsável pelo equilíbrio térmico.

As Lesões por Pressão (LPP) correspondem a uma série de alterações ocorridas em alguma camada da pele resultantes de uma pressão excessiva em áreas de proeminência óssea, na qual podem ser decorrentes de fricção do paciente a beira leito, cisalhamento ou por pacientes cadeirantes devido à longa permanência em uma mesma posição. Esses tecidos passam por constantes transformações tanto por processos fisiológicos, quanto por modificações patológicas que podem ser desencadeadas por fatores intrínsecos ao paciente como desidratação, déficit nutricional, envelhecimento e/ou fatores extrínsecos ao paciente como a pressão que é exercida sobre o tecido, não mudança de decúbito e umidade da pele causada por suor ou incontinência (Oliveira et al., 2019).

Segundo a epidemiologia das LP, estudos internacionais apontam que a taxa de incidência para o desenvolvimento das lesões são de aproximadamente $3,51 \%$ a $25,9 \%$ tendo uma prevalência de $16,9 \%$ a 23,8\% em pacientes hospitalizados em 
regime de terapia intensiva. Em unidades clínica, a incidência e de 1,8\% e uma prevalência de 3,3\% os pacientes internados. No Brasil, a incidência de LP em pacientes hospitalizados em unidade de terapia intensiva e de 13,95\% e a prevalência de 17,99\%, já em unidades clinicas e cirúrgicas tem um prevalência de que cerca de $10 \%$ dos pacientes admitidos desenvolvem LP (Santos et al,2020).

Ao se avaliar o risco de um paciente desenvolver LP, diversos fatores de risco devem ser levados em consideração. Para direcionar esta avaliação utiliza-se de instrumentos específicos e comprovados cientificamente, como a Escala de Braden. O paciente pode ser classificado em quatro tipos, a saber: (1) como sem risco; (2) com risco baixo, (3) moderado ou (4) alto, de acordo com o escore obtido nas sub escalas: percepção sensorial, umidade, atividade, mobilidade, nutrição, e friç̧ão ou cisalhamento (Rodrigues et al., 2011). Diante disso, o profissional deve ater-se a todas as condições do paciente e coletar todas as informações necessárias durante exame físico, entrevistas e em até mesmo em procedimentos diários como banho no leito, pois irão contribuir significativamente para identificar precocemente as injurias.

De acordo com Souza et al. (2017), pessoas idosas são mais suscetíveis a desenvolverem lesão por pressão devido a condições do envelhecimento humano, como a uma diminuição da espessura da pele, das fibras elásticas, rigidez do colágeno e diminuição de capilares, que ocasionam uma redução do suprimento sanguíneo e desidratação da pele. Além do mais, os autores citam ainda que o aparecimento de doenças-crônicas degenerativas comuns nessa fase da vida podem causar restrições motoras, visuais intelectuais e auditivas em pessoas com mais idade, reduzindo sua condição de vida. Dessa maneira, o enfermeiro deve atentar - se rigorosamente aos cuidados prestados a esses pacientes, visto aos inúmeros agravantes.

Além dos idosos por conta da vulnerabilidade de aspectos do envelhecimento, as LPP's também são uma complicação grave e frequente em pacientes com lesão medular, interferindo direta ou indiretamente no bem-estar dessas pessoas. Quando acometidas por lesões teciduais de níveis mais altos, tornam- se ainda mais rigorosos os cuidados devido á dificuldade de cicatrização, requerendo-se uma maior frequência de visitas aos serviços de saúde, refletindo assim, em dificuldades relacionadas a deslocamento e questões financeiras do paciente (Sousa et al., 2017).

Segundo Almeida et al. (2019, p. 01), “[...] pessoas com feridas [...] enfrentam alterações na imagem corporal, prejuízos na mobilidade deficit no autocuidado, incapacidade para realização das atividades de vida diária, presença de dor e de desconforto que acarretam impactos negativos na qualidade de vida". Desse modo, a aceitação por parte desses pacientes influência diretamente em seu tratamento, uma vez, que o entendimento da sua condição corporal contribui positivamente para aceitação tendo uma boa evolução clínica.

Ademais, a pele é um "cartão" de visita dos (a) indivíduos (a), pois através dela podemos sentir e expressar inúmeras sensações, por exemplo, alterações na linguagem corporal como o rubor da face/rosto ao se envergonhar e/ou arrepios sentidos em fontes emoções e uma forma de expressar- mos o que sentimos e quem somos. A partir deste pressuposto e levando em consideração a sua importância pacientes que desenvolvem alterações significativas na pele tendem a ter uma redução na qualidade de vida, seja através de desenvolvimento de quadros de insegurança, medo, vergonha, desejo pelo isolamento e mudanças em suas relações de convívio social.

Nesse contexto, o presente trabalho visa fazer uma reflexão teórica a respeito da qualidade de vida de pacientes que apresentam lesão tecidual, buscando também avaliar os fatores que corroboram para o surgimento dessas alterações no tecido, mostrar quais são os estágios da lesão e suas consequências e refletir sobre a percepção corpórea do paciente diante dessas alterações.

\section{Metodologia}

Por meio de uma pesquisa qualitativa e de procedimento bibliográfico (Gil, 2011), investigou-se em artigos científicos que tratam da temática em questão. De posse desse material, realizou-se as leituras que possibilitou apropriar-se ao tema de 
pesquisa seguido as seguintes etapas:(1) elaboração da questão norteadora;(2) estabelecimento de critérios para inclusão e exclusão dos artigos;(3) definição das informações a serem extraídas de cada estudo;(4) avaliação dos incluídos na temática;(5) interpretação dos resultados.

A questão norteadora do escrito foi "O que a literatura evidencia sobre a Qualidade de Vida em Pacientes Portadores de Lesão Por Pressão" a seleção bibliográfica para compor o estudo deu-se início em janeiro de 2021 através dos indicadores online, que se encontram referenciados no sistema de busca plataforma Google Acadêmico, Literatura Latino Americana e do Caribe em Ciências da Saúde (LILACS) e SCIELO.

A busca pelos estudos foi feita pelos pesquisadores supracitados de modo independente considerando como critério de inclusão estudos no período de 2010 a 2021, artigos que abordem qualidade de vida. Para realização das buscas foram utilizados combinações entre as seguintes palavras chaves: Lesão Por Pressão; Qualidade de Vida; Cuidados de Enfermagem e Feridas Crônicas. No idioma português e inglês sendo eles artigos de ensaios clínicos randomizados, texto completo, estudo transversal, estudos de coorte prospectivos, estudo descritivo e revisões integrativas.

Os critérios para exclusão foram artigos fora da temática, documentos incompletos, monografias, revisões bibliográficas e artigos duplicados. Foi feito uma análise exploratória dos resumos, sendo encontrados o total de 38 artigos na base de dados Google Acadêmico e 18 na base de dados LILACS e 7 na SCIELO foram selecionados para ler, após a leitura criteriosa 6 foram escolhidos como objeto de estudo.

\section{Resultados}

Os trabalhos analisados para construção da pesquisa são os seguintes: Úlcera por pressão após a alta hospitalar e o cuidado em domicílio (Jaísa Valéria Moro e Maria Helena Larcher Caliri); Fatores associados à incidência da úlcera por pressão durante a internação hospitalares (Fernanda Penido Matozinhos); Qualidade de vida em portadores de lesão medular com úlceras por pressão (Dalete Mota e Milainy Barbosa Ribeiro); Analisar a produção científica sobre a assistência de enfermagem com vista à prevenção de lesão por pressão em pacientes hospitalizados (Francinalva de Almeida; Maria dos Milagres Santos da Costa; Ellen Eduarda Santos Ribeiro; Danielle Christina de Oliveira Santos; Nara Daniele Alcântara Silva; Rosilda Evangelista da Silva; Kamilla Pinheiro Saraiva; Polyana Coutinho Bento Pereira; Incidência de lesões de pele, risco e características clínicas de pacientes críticos ( Dandara Soares Monteiro; Eline Lima Borges; Josimare Aparecida Otoni Spira; Taysa de Fátima Garcia; Selme Silqueira de Matos); A influência das lesões por pressão na qualidade de vida e inclusão dos usuários de cadeira de rodas ( Bruna Henkel Ferro; Jacinta Sidegum Renner; Michele Barth; Eliane Fátima Manfio). Segue a tabelas com os resultados (1), posteriormente a tabela (2) com ano, local de publicação e metodologia: 
Tabela 1: Análise variada de artigos com relação da presença de lesões por pressão abordando tratamento e as variáveis da doença. Teresina (PI). 2021.

\begin{tabular}{|c|}
\hline Resultados \\
\hline $\begin{array}{l}\text { Dos } 21 \text { artigos analisados houve predominância de risco para desenvolvimento das LP no sexo feminino, e em } 9 \text { artigos a variável avanço da } \\
\text { idade estava relacionada aos fatores de risco, visto que o envelhecimento traz modificações na pele, baixa oxigenação dos tecidos, redução } \\
\text { de nutrientes e outros fatores que contribuem para uma maior vulnerabilidade dos senis a sofrerem diversos tipos de lesões. Nove associam o } \\
\text { uso de medicamentos com a ocorrência de LP nos idosos, pois existem algumas medicações que podem influenciar no processo de } \\
\text { cicatrização das lesões. }\end{array}$ \\
\hline $\begin{array}{l}\text { A amostra foi composta de } 442 \text { alunos e a mediana de permanência de internação hospitalar foi de } 22 \text { dias (IQ=16- } 35) \text { Foram registrados } 25 \\
\text { casos incidentes de LP, sendo } 10.324 \text { o número de pacientes- dia. O estudo verificou que a mediana do IMC foi de } 23,44 \mathrm{~kg} / \mathrm{m} 2(\mathrm{IQ}=20,76- \\
27,34) \text {. Observou- se o predomínio de indivíduos com faixa etária entre } 18 \text { e } 60 \text { anos }(27,5 \text { ou } 62,22 \%) \text {, do sexo feminino }(24,3 \text { ou } 54,98 \%) \\
\text {, da cor de pele parda e negra }(22,3 \text { ou } 51,15 \%) \text {, não tabagistas }(22,5 \text { ou } 51,96 \%) \text {, sem risco, conforme escores originais da escala de Braden } \\
(26,5 \text { ou } 59,95 \%) \text {, e de eutróficos }(17,9 \text { ou } 49,04 \%) \text {. }\end{array}$ \\
\hline $\begin{array}{l}\text { Dos } 118 \text { portadores de traumatismos da medula espinhal internados no período de coleta de dados, } 58(49,1 \%) \text { apresentaram lesões por } \\
\text { pressão. Dentre esses, } 40 \text { responderam aos instrumentos. A análise do WHOQOL-bref evidenciou escore muito baixo no Domínio físico } \\
(53,04) \text {, seguido dos domínios psicológico }(60,94) \text { e ambiental }(63,52) \text {. O único domínio com escore satisfatório foi o social (70,21). De } \\
\text { modo geral os escores se apresentaram preocupantes. A avaliação da qualidade de vida elucida uma insatisfação significativa dos } \\
\text { participantes, principalmente quanto aos aspectos físicos relacionadas ao trauma e o surgimento de lesões por pressão. }\end{array}$ \\
\hline $\begin{array}{l}\text { Os resultados evidenciaram a importância de padronizar as notificações e qualificar a equipe de Enfermagem para atuar de forma mais eficaz } \\
\text { na intervenção e prevenção das lesões por pressão. }\end{array}$ \\
\hline $\begin{array}{l}\text { Em uma pesquisa qualitativa realizada com um grupo de } 22 \text { pessoas usuárias de cadeira de rodas da Associação dos Lesados Medulares do } \\
\text { Rio Grande do Sul (LEME), os resultados indicaram que o mais recorrente problema mencionado foi o medo da morte em decorrência das } \\
\text { lesões e a dependência do cuidador, que limita seu cotidiano em funções dos curativos, do mau cheiro emanado das injurias e dos } \\
\text { constrangimentos físicos e emocionais. }\end{array}$ \\
\hline $\begin{array}{l}\text { tudo de coorte retrospectivo realizado em centro de terapia intensiva com amostra de } 125 \text { pacientes cujo des } \\
\text { resultantes a nutrição enteral foi um preditor para o desenvolvimento de algum tipo de lesão. Obteve que os }\end{array}$ \\
\hline
\end{tabular}

Fonte: Pesquisa própria.

Tabela 2: Ano, local de publicação e metodologia dos trabalhos analisados Teresina (PI). 2021.

\begin{tabular}{|l|l|l|}
\hline Ano & Local de publicação & Metodologia de pesquisa \\
\hline 2016 & Escola Anna Nery & Estudo transversal com abordagem quantitativa \\
\hline 2017 & $\begin{array}{l}\text { Revista da Escola de } \\
\text { Enfermagem da USP }\end{array}$ & Estudo de coorte prospective \\
\hline 2016 & Lectrónica Enfermería Global & Estudo descritivo, transversal, com abordagem quantitativa \\
\hline 2020 & $\begin{array}{l}\text { Revista Eletrônica Acervo } \\
\text { Saúde }\end{array}$ & Revisão integrativa de literature \\
\hline 2021 & $\begin{array}{l}\text { Revista Interdisciplinar de } \\
\text { Estudos em Saúde da } \\
\text { UNIARP }\end{array}$ & Estudo qualitative \\
\hline & $\begin{array}{l}\text { Texto e Contexto } \\
\text { Enfermagem }\end{array}$ & Estudo de coorte retrospectivo \\
\hline
\end{tabular}

Fonte: Pesquisa Própria.

\section{Discussão}

\section{Estágios da Lesão por Pressão e suas Consequências}

Os estágios da LPP irão influenciar diretamente em seu tratamento desde o uso da Terapia Tópica Escolhida, isto é, os medicamentos que são utilizados sobre a pele lesionada. É importante ressaltar, que não apenas à Terapia Tópica terá garantias de um bom resultado da lesão, outros fatores devem ser levados em consideração tais como: boa oxigenação do paciente; doenças crônicas associadas como (hipertensão arterial, diabetes mellitus) (Araujo,2019). Segundo Sistema de Classificação da Nacional Pressure Ulcer Advidory Panel (NPUAP, 2016), essas lesões podem ser: 
Estágio 1: Pele íntegra com eritema (...) - Pele integra com área localizada de eritema (...). Estagio 2: Perda da pele em sua espessura parcial com exposição da derme- Há a ruptura da epiderme e exposição da derme, leito da ferida é viável e pode apresentar- se como bolha intacta ou rompida, não tem tecido de granulação. Estágio 3: Perda da pele em sua espessura total - Neste estágio a pele se rompe em sua espessura total e gordura fica visível. Estágio 4: Perda da pele em sua espessura total e perda tissular- (...), na qual há exposição ou palpação direta de estruturas como fáscia, tendão, cartilagem e osso. (...) Lesão por Pressão Não Classificável: Perda da pele em sua espessura total e perda tissular não visível por está encoberto de esfacelo ou escara, dificultando identificar profundidade do dano. Há hiperemia e endurecimento em bordas.

O estágio na qual se encontra a lesão possuí relação direta na execução das atividades da vida diária a depender do nível que se apresentar a LPP, os pacientes tendem a desenvolver um grau de dependência e restrições maiores, desde a não mudança de decúbito, fator essencial para prevenção e piora da lesão. O agravamento pode levar a quadros de osteomielite, septicemia, diminuição da autoestima, isolamento social e transtorno psicológicos, uma vez que, o odor exalado pela ferida torna-se perceptível a outras pessoas (Machado, 2018)

Assim como os estágios de classificação da lesão, é possível identificar que a localização da LPP também configura um fator importante por auxiliar no direcionamento do tratamento, dado que a cada situação faz- se necessária uma abordagem de curativos e cuidados específicos. Os locais de ocorrência das lesões são: região sacral, occipital, trocantes e calcâneas. Além do desconforto e sofrimento vivenciados pelo paciente durante sua hospitalização, seu tratamento constitui um gasto financeiro muito maior que a prevenção da LPP, pois há aumento de custos na estadia dos pacientes nos hospitais e os submetem a um maior risco de infecções hospitalares fazendo- se necessário a utilização de ainda mais recursos como melhores coberturas, curativos, colchões especiais, almofadas, coxins ou espumas para redistribuição da pressão exercida no corpo.

Observando o trabalho de Rodrigues (2011) feito na região do arquipélago dos Açores constatou-se que 99 enfermeiros (a) domiciliares e 13 enfermeiros responsáveis por outras instituições de saúde, nesta pesquisa objetivou a coleta de dados sobre os fatores que influenciavam os cuidados domiciliares de prevenção em pacientes com risco para LPP e obteve os seguintes resultados: os principais fatores advêm da falta de recursos materiais $(43,4 \%)$ e de recursos humanos $(30,3 \%)$. Dessa forma, é importante que o enfermeiro (a) tenha um bom raciocínio clínico no planejamento de ações que se adequem a cada quadro, visto as diversas condições socioeconômicas vividas por cada paciente, bem como valores morais, culturais, crenças e fatores comportamentais que podem influenciar diretamente na qualidade da assistência prestada a este.

Além disso, medidas preventivas são de fundamental importância, pois reduzem a taxa de morbidade e mortalidade com ações como a redistribuição da pressão corporal, a mudança de decúbito de no máximo a cada duas horas contando com a participação multiprofissional dos serviços de saúde e a participação da família com a identificação daqueles que apresentam serem mais propensos a ocorrência de LPP como (idosos e obesos) (Pacha et al., 2018).

\section{Qualidade de Vida dos Pacientes com Lesões por Pressão}

Refletir sobre qualidade de vida dos (a) pacientes com lesões por pressão, bem como as feridas que causam dor e desequilíbrios emocionais, o risco de desenvolver infecções, perda da funcionalidade ou afastamento do trabalho, ocasionando prejuízos financeiros e custos com tratamentos para o Ministério da Saúde e para o (a) paciente (Portugal et al., 2018). Nesse sentido, deve-se sempre levar em questão o princípio da integralidade, buscando formar apoio em rede de serviços do Sistema Único de Saúde - SUS que deverão dar suporte a estes objetivando o cuidado em relação aos aspectos supracitados que podem ser afetados. 
Quando esse indicador é debatido sobre as (LP), geralmente acabamos associando somente a população mais idosa, porém outro grupo a manter- se constante vigilância quanto aos riscos devidos esses danos, são os indivíduos que utilizam cadeiras de rodas, devido ao longo tempo que geralmente ficam na posição sentada, sofrendo constante cisalhamento e pressão com a superfície do equipamento de locomoção.

Em pesquisa trabalhada em uma instituição filantrópica, a Associação dos Lesados Medulares do Rio Grande do Sul com 22 pessoas usuárias de cadeias de rodas, mostrou que o medo das LPP's permeiam toda à rotina dessas pessoas e que é expresso por elas de forma contundente como sendo uma questão limitadora na sua qualidade de vida, visto que ao mesmo tempo que o objeto de locomoção oportuniza o direito de ir e vir torna possível uma vida social mais plena, o mesmo acaba propiciando risco e temor pela abertura de feridas, do risco de infecção e mau cheiro emanado nesses locais e trazem constrangimento tanto físico quanto moral que limitam sua independência e sua autonomia, interferindo na vida social, familiar e do trabalho (Ferro et al., 2021).

Um estudo realizado por Moro e Caliri em domicílios com pacientes adultos e idosos, em 2016 no Município de Ribeirão Preto/SP verificou que 69,5\% dos pacientes analisados referiram terem tido orientação sobre o uso de medidas e prevenção de LPP. Entretanto, verificando-se que essas orientações eram focadas somente em medidas tradicionais como hidratação da pele e mudança de decúbito, e que não supriam as necessidades dos pacientes de informações sobre ações de manejos para os problemas que os colocavam para risco de lesões.

Sendo assim, a falta de conhecimento ocasionada por orientações e informações insuficientes dadas pelos profissionais de saúde aos familiares e/ou cuidador contribuem para que haja redução do bem estar do paciente e o agravamento de suas lesões. À vista disso, não há um direcionamento aos clientes que os preparam as situações após a alta hospitalar como, por exemplo, identificação de sinais de infecção das feridas, higiene, hidratação da pele e mudanças de posição.

Nesta perspectiva, os autores Almeida (et al., 2019) consideram que o aumento do conhecimento por parte da equipe de Enfermagem e a implementação de práticas baseadas em evidências científicas trazem benefícios tanto na redução do tempo de internação hospitalar quanto na diminuição da incidência de casos. Ademais, com toda a capacitação para lidar com o aparato tecnológico e a busca por ações que foquem em formas de tratamento eficazes aos diversos estágios e regiões das lesões, assim o planejamento desses cuidados fazem com que os clientes ganhem mais qualidade de vida.

Diante do referido, as ações de saúde e principalmente da equipe de Enfermagem que lidam diretamente com esses cuidados específicos, devem ser voltados para terapêutica educacional orientando o próprio indivíduo, família e/ou cuidador sobre formas de como minimizar riscos não só no hospital, como também em casas de repouso e domicílio. Uma pesquisa realizada com pacientes internados no setor da unidade intensiva do hospital universitário do Ceará a respeito da contribuição da tecnologia educacional sobre lesões por pressão em indicadores de qualidade assistenciais obteve como resultados que o número de pacientes com lesão por pressão diminuiu de 53,2\% para 42,6\% depois de dadas intervenções educacionais, concluiu-se de forma positiva o impacto da tecnologia de informação e comunicação principalmente na incidência de lesões (Caldini et al., 2018)

O estado nutricional também é um fator de extrema importância na prevenção de danos à pele, especialmente LPP' s. A deficiência nutricional afeta diretamente a tolerância do tecido, propriedades mecânicas da pele, morfologia do tecido e propriedades fisiológicas, como reparo e propriedades térmicas (Monteiro et al., 2021). Uma boa nutrição ira influenciar diretamente na evolução positiva da ferida, pois a partir dela se fornece os macros e os micro nutrientes que são necessários para o bom funcionamento do organismo resultando em uma melhor evolução do quadro clinico. Esse fator é um essencial cooperante no tratamento dessas lesões cutâneas, visto que principalmente pacientes que são alimentados por via sonda enteral 
já possuem esse processo prejudicado e necessitam de um suporte e atenção maior dos profissionais para se evitar quadros de desidratação e desnutrição prejudicando assim a melhora do cliente. (Munoz et al.,2020).

Dessa forma, a equipe então deve utilizar os protocolos, práticos que se baseiam em evidências, para exercer uma boa comunicação com linguagem clara e objetiva buscando sempre suprir os questionamentos elencados acima, além de saber identificar as reais carências individuais, pois são todos os quesitos relevantes que trazem segurança ao paciente para uma recuperação e número reduzido de incidência das LPP.

\section{Considerações Finais}

Objetivou-se no presente trabalho pontuar os fatores que corrobora para o surgimento das lesões por pressão junto de seus agravos, bem como as consequências na saúde do cliente, assim nosso objetivo foi alcançado, sendo possível refletir sobre a qualidade de vida em pacientes portadores de lesão por pressão. Desse modo, por meio da revisão da literatura com sustentação dos (a) autores, estes demonstraram que as informações são a base para eficácia da assistência de cuidados, pois é necessário que a equipe multiprofissional esteja capacitada através da constante atualização de conhecimentos técnicos, científicos e tecnológicos, para que deste modo possa repassar com objetividade e clareza ao paciente a prevenção e seu autocuidado. Contudo, ainda são vastas as lacunas existentes a respeito das reais dificuldades enfrentadas por indivíduos com lesão por pressão e sua qualidade de vida. Sendo assim, se faz necessária o aprofundamento de pesquisas relacionadas à questão para contribuir em melhores resultados.

\section{Referências}

Ascari, R. A. et al. (2014) Úlcera por pressão: um desafio para a enfermagem. Brazilian Journal of Surgery and Clinical Research, 6(1), 11-16. https://www.mastereditora.com.br/periodico/20140301_132755.pdf

Almeida, F., Costa, M. M. S., Ribeiro, E. E. S., Santos, D. C. O., Silva, N. D. A., Silva, R. E., Saraiva, K. P., \& Pereira, P. C. B. (2019) Assistência de enfermagem na prevenção da lesão por pressão: uma revisão integrativa. Revista eletrônica acervo em saúde. https://doi.org/10.25248/reas.e1440.2019.

Almeida, S. et al. (2019). Depressão em pacientes com lesões traumáticas da medula espinhal e úlceras de pressão. Revista Brasileira de Cirurgia Plástica, 28(2), 282-288, https://doi.org/10.1590/S1983-51752013000200019.

Araujo, M. T. et al. (2019). Análise de custo da prevenção e do tratamento de lesão por pressão: revisão sistemática. Revista Enfermagem Atual In Derme, 89(27). https://revistaenfermagematual.com/index.php/revista/article/view/47.

Caldini, L. N. et al. (2018) Avaliação de tecnologia educativa sobre lesão por pressão baseada em indicadores de qualidade assistenciais https://pesquisa.bvsalud.org/portal/resource/pt/biblio-947609.

Ferro, J. S. R. et al. (2021). A influência das lesões por pressão na qualidade de vida e inclusão social: a percepção dos usuários de cadeiras de rodas. Revista Interdisciplinar de Estudos em Saúde da UNIARP. https://doi.org/10.33362/ries.v9i1.1739.

Gil, A.C. (2011). Métodos e Técnicas de Pesquisa social. (6a ed.), Atlas,

Machado, D. O. et al. (2018). Cicatrização de lesões por pressão em pacientes acompanhados por um serviço de atenção domiciliar. Texto \& ContextoEnfermagem, 27(2). https://www.scielo.br/scielo.php?pid=S0104-07072018000200329\&script=sci_abstract\&tlng=pt.

Moro, J. V., \& Caliri, M. H. L. (2016). Úlcera por pressão após a alta hospitalar e o cuidado em domicílio. Escola Anna Nery, 20(3). https://pesquisa.bvsalud.org/portal/resource/pt/lil-783892.

Monteiro, D. S. et al. (2021). Incidência de lesões pele, risco e características clínicas de pacientes críticos. Texto \& Contexto - Enfermagem [online]. 30, e20200125. https://doi.org/10.1590/1980-265X-TCE-2020-0125.

Mota, D. R. et al. (2016). Qualidade de vida em portadores de lesão medular com úlceras. Enfermaria Global. 5, 13-21. http://scielo.isciii.es/scielo.php?script=sci_arttext\&pid=S1695-61412016000200002\&lang=pt.

Macedo, F. P. et al. (2018). Fatores associados a incidência de úlcera por pressão durante a internação hospitalar. Revista da Escola de Enfermagem da USP.51. https://doi.org/10.1590/s1980-220x2016015803223.

Munoz, N. et al. (2020). O papel da nutrição na prevenção e cura de lesões por pressão a recomendação da diretriz de pratica clinica internacional de 2019. Clinical Monagement Extra.33, 123-136. https://journals.1ww.com/aswcjournal/Fulltext/2020/03000/The_Role_of_Nutrition_for_Pressure_Injury.3.aspx.

National Pressure Ulcer Advisory Panel (NPUAP). (2016). Revista de Enfermagem do Centro Oeste Mineiro, 6(2). 10.19175/recom.v6i2.1423. 
Research, Society and Development, v. 10, n. 17, e233101724391, 2021

(CC BY 4.0) | ISSN 2525-3409 | DOI: http://dx.doi.org/10.33448/rsd-v10i17.24391

Oliveira, A. C et al. (2019). Qualidade de vida de pessoas com feridas crônicas. Acta Paulista de enfermagem, 32(2), 194-201. https://www.scielo.br/scielo.php?script=sci_arttext\&pid=S0103-21002019000200194.

Pachá, H. H. P et al. (2018). Lesão por Pressão em Unidade de Terapia Intensiva: estudo de caso-controle. Revista Brasileira de Enfermagem, 71(6), 30273034. https://www.scielo.br/scielo.php?pid=S0034-71672018000603027\&script=sci_arttext\&tlng=pt.

Portugal, L. B. A., \& Christovam, B. P. (2018). Estimativa do Custo do Tratamento da Lesão por Pressão, Como Prevenir e Economizar Recursos. Revista Enfermagem Atual In Derme, 86(24). https://revistaenfermagematual.com.br/index.php/revista/article/view/93/18.

Rodrigues, A. M., Soriano, J. V. (2011). Fatores influenciadores dos cuidados de enfermagem domiciliários na prevenção de úlceras por pressão. Revista de Enfermagem Referência, (5), 55-63. http://dx.doi.org/10.12707/RIII1119

Souza, N. R et al. (2017). Fatores predisponentes para o desenvolvimento da lesão por pressão em pacientes idosos: uma revisão integrativa. Estima-Brazilian Journal of Enterostomal Therapy, 15(4). https://www.revistaestima.com.br/estima/article/view/442.

Sousa, S. A. M., da Silva Pires, P., Macedo, M. P., Oliveira, L. S., Batista, J. E. T., \& Amaral, J. M. (2017). Lesão por pressão: incidência em unidades críticas de um hospital regional. https://pdfs.semanticscholar.org/4d1f/99ab60e81743744cc34b05596fa40eaac948.pdf .

Santos, C. T et al. (2020). Indicadores da nursing autocomes classification para avaliação de pacientes com lesão por pressão: concenso de especialistas. Escola Anna Nery, 25(5). https://www.scielo.br/j/ean/a/8t8rjTqvJKgX3rFbCDMkXWb/?lang=pt. 